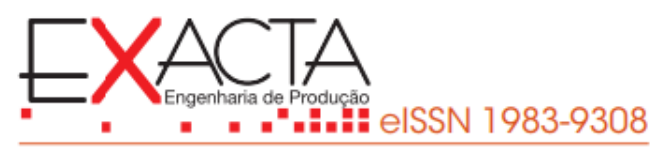

\title{
ANÁLISIS Y MAPEO DEL PROCESO PRODUCTIVO EN UNA CONCESIONARIA DE MINIBUSES
}

\author{
ANALYSIS AND MAPPING THE PRODUCTION PROCESS AT A MINIBUSES \\ DEALERSHIP
}

Recebido em: 08 mar. 2019

Aprovado em: 11 mar. 2020

Versão do autor aceita publicada online: 11 mar. 2020

Publicado online: 12 maio 2021

\section{Como citar esse artigo - American Psychological Association (APA):}

Silva, R. N. A., Silva, S. M. O., Farias, F. A. de S., Cunha, S. T., \& Astuto, M. da S. (2021, out./dez.). Análisis y mapeo del proceso productivo en una concesionaria de minibuses. Exacta. 19(4), 745-763. https://doi.org/10.5585/exactaep.2021.13213.

Submeta seu artigo para este periódico $\beta$

Dados Crossmark 


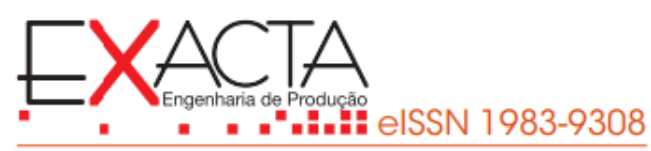

\title{
ANÁLISIS Y MAPEO DEL PROCESO PRODUCTIVO EN UNA CONCESIONARIA DE MINIBUSES
}

\author{
ANALYSIS AND MAPPING THE PRODUCTION PROCESS AT A MINIBUSES \\ DEALERSHIP
}

Raimundo Nonato Alves da Silva ${ }^{1}$

Sônia Maria Oliveira e Silva ${ }^{2}$

Francisco Assis de Souza de Farias ${ }^{3}$

Stefanny Terencio da Cunha ${ }^{4}$

Marcello da Silva Astuto

1 PhD student at Metallurgical and Materials Engineering.

University of State of Amazonas - UEA.

Faculty of Engineering of University of Porto - FEUP.

Porto - Portugal.

raimundo.nonato.silva@gmail.com

2 Doutora em Língua Portuguesa.

Faculdade Martha Falcão - FMF.

Manaus, Amazonas - Brasil.

soniaoliveira-mao@hotmail.com

${ }^{3}$ Especialista em Administração Empresarial.

Centro Universitário do Norte - UNINORTE.

Manaus, Amazonas - Brasil.

francisco@ffgestaoempresarial.com.br

${ }^{4}$ Engenheira de Produção.

Centro Universitário do Norte - UNINORTE.

Manaus, Amazonas - Brasil.

stefannyterencio.mx@gmail.com

${ }^{5}$ Mestre em Gestão Empresarial.

Centro Universitário de Ensino Superior do Amazonas - CIESA.

Manaus, Amazonas - Brasil.

marcello.astuto@gmail.com

Recebido em: 08 mar. 2019

Aprovado em: 11 mar. 2020
Resumen: El servicio al cliente o incluso la calidad del servicio hoy en día se traduce como una de las principales preocupaciones de las empresas modernas, con respecto a la continuidad o incluso el aumento de la competitividad que se puede entender como la satisfacción del cliente. Esta investigación tiene como objetivo analizar posibles "lagunas" involucradas en la realización del servicio en procedimientos de mantenimiento preventivo y correctivo aplicados en el minibús, en un concesionario que involucra el taller y el almacén de piezas utilizando diagramas de flujo para procesos de mapeo. El método de investigación fue adoptado como un estudio de caso. Los datos se recopilaron a través de entrevistas y se usaron posteriormente en la preparación de diagramas de flujo, para que pudieran conducir procesos de mapeo. Evaluamos los resultados, encontramos lagunas en la aplicación de diagramas de flujo en el mapeo de procesos tales como fallas en el servicio de su tipo para llevar a cabo, entrega, recolección inadecuada de mantenimiento, falta de material en la tienda, demora entre la solicitud y confirmación para la realización de servicios, entre otros. El resultado es, luego, la eliminación de las lagunas con la implementación y el monitoreo de los indicadores de desempeño en los procesos, además de los elementos considerados más críticos para la concesionaria.

Palabras clave: Mapeo. Diagrama de flujo. Concesionaria. Gestión de procesos. Manaus.

Abstract: The customer service or even service quality today translates as a major concern of modern enterprises, with regard to continuity or even increasing competitiveness that can be understand as customer satisfaction. This research has as objective to analyze possible "gaps" involved in making the service in preventive and corrective maintenance procedures applied in the minibus, in a dealership, involving the workshop and parts store using flowcharts for mapping processes. The research method was adopted as a case study. The data were collected through interviews and subsequently used in the preparation of flowcharts, so they could drive mapping processes. Evaluating the results, we found gaps in the application of flowcharts in the mapping of processes such as failures in the service of its kind to carried out, delivery, inadequate collection of maintenance, lack of material in store, delay between the request and confirmation for the performance of services, among others. Result is, then the elimination of gaps with the implementation and monitoring of performance indicators in the processes, in addition to the items considered most critical to the dealership.

Keywords: Mapping. Flowchart. Dealership. Management processes. Manaus. 


\section{Introducción}

Debido al crecimiento de la población en los grandes centros urbanos y la popularización de las tecnologías, las empresas de servicios vienen ganando destaques en la economía del país, y en muchos casos son los buques insignia en valores do Producto Interno Bruto (PIB). En Brasil, segundos datos de (IBEGE, 2017), e de acuerdo con los resultados del Sistema de Cuentas Nacionales, la participación de los servicios atingió $68,5 \%$ del PIB del país.

El segmento de servicios de ventas de minibús, partes y piezas, objeto de esta investigación, es considerada agresiva en relación con la concurrencia. En este escenario, surge la necesidad de algunas empresas reinventaren sus procesos, como una estrategia para aprovechar sus facturas.

Asegura (Slack, Chambers, Johnston, \& Betts, 2013), que la importancia estratégica de operaciones y de procesos es cada vez más reconocida. En otras palabras, las operaciones son recursos que producen productos y servicios; en cuanto el operacional es el opuesto de estratégico; significa detallado, localizado, curto plazo y cotidiano (Slack et al., 2013). Estas dos vertientes van a orientar este trabajo a donde la investigación por la gerenciarían de las operaciones y de procesos presentan impacto significativo en la estrategia de la empresa. A través de un eficiente mapeo de los procesos a lo largo de los departamentos de la organización, la investigación mostrará las oportunidades de mejorías con análisis efectivas de los servicios prestados.

Las mejorías (Davenport \& Prusak, 2003) en los procesos son representativas para el resultado de la empresa. Sugiere el autor enfocar en los procesos clave, que deben ser reajustado para satisfacer múltiples objetivos de negocio, entre ellos la reducción de tiempos de los procesos.

El objetivo de este estudio es analizar posibles "gaps" implicados en la realización del servicio en los procesos de mantenimiento preventivo y correctivo aplicados en los minibuses, envolviendo el taller y la tienda de piezas con el uso da herramienta de mapeo de procesos de acuerdo con los procesos de ingeniería de manufactura.

Para que este objetivo sea alcanzado, serán necesarios que se atínjanlos siguientes objetivos específicos: analizarlos procesos de los servicios del taller y tienda de piezas de la concesionaria; identificar puntos vulnerables para la realización de los servicios en las empresas; e indicar posibles mejorías para aumentar la eficiencia de los servicios.

El uso de los minibuses en las grandes capitales deja de ser un modismo para mostrarse como una verdadera utilidad en la movilidad de la clase media y media alta con acceso a un coche, más dispuesta a dejarlo en casa para usar un transporte colectivo con mínimo conforto y una relación diferenciada de costo beneficio.

Si es así, los servicios de mantenimiento preventivo o correctivo, desenvueltos en una concesionaria de vehículos, deben en su esencia posibilitar a los clientes rapidez aliada a calidad de los 
servicios. Por otro lado, satisfacerlas necesidades de los clientes y garantir el retorno financiero para el propietario de la empresa prestadora de lo servicio.

Entonces, como relevancia, para el análisis de este ambiente en una empresa de servicios, serán aplicadas herramientas de mapeo y gestión de procesos, posibilitando analizar científicamente posibles soluciones con bajo investimentos.

\section{Materiales y métodos}

Este estudio tuvo su enfoque en una empresa localizada en Manaus, Amazonas, que comercializa vehículos, piezas y presta asistencia técnica especializada en minibuses, ofreciendo todo el suporte, con foco en los sectores de transporte colectivos alternativos y transporte de funcionarios para las industrias PIM (Polo Industrial de Manaus) y otros segmentos.

El método de investigación fue adoptado como un estudio de caso. Este método investiga y presupone la preservación de las características de los acontecimientos de la realidad actual, como los ciclos de vida individuales, procesos organizacionales y administrativos, cambios en regiones urbanas, relaciones internacionales y la maduración de algunos sectores. El estudio de caso se ocupa de múltiples fuentes de evidencia, investigando un tópico empírico según procedimientos preespecificado. El objeto de un estudio de caso es una unidad que se analiza profundamente (Triviños, 2013; Yin \& Grassi, 2010). Este trabajo adoptó el tipo de investigación exploratoria y descriptiva. Que de acuerdo (Gil, 2017), se desarrollan para proporcionar una visión general, de tipo aproximativo, acerca de determinado hecho. Los procesos en esta empresa son diversos y los elegidos para esta investigación se refieren tan sólo al mantenimiento (preventivo y correctivo) de los microbuses donde demostraron mayor fragilidad en los procesos de los servicios. Los primeros pasos de este estudio fueron comprender las fases de selección de los objetivos estratégicos de referencia, selección de los factores clave, selección de los procesos relacionados con los factores claves y selección de procesos prioritarios. Estas fases pueden ser así detalladas: -Selección de los objetivos estratégicos de referencia: corresponde al establecimiento de los resultados deseados para el negocio, derivados del análisis de la misión de la empresa, del plan estratégico y del escenario del mercado. -Selección de los factores clave: corresponde al conjunto de condicionantes / variables importantes para el éxito de la organización en la realización de sus objetivos de referencia (Miyashita \& Salomom, 2011). En seguida, se produjo una evaluación del proceso de la empresa de servicios en minibús a través de un levantamiento documental, observaciones y entrevistas conforme apéndices con algunos participantes en el proceso.

O estudio se limita a la satisfacción de los clientes de la concesionaria en relación con los gaps existentes en los procesos de los servicios que la empresa ofrece, ya que la satisfacción es una consecuencia de varios factores que no son objetos de éste. En relación con la utilidad de los procesos 
mapeados éstos se tornaron esenciales en la identificación de los procesos vitales de la organización, además de objetivar entender y comunicar el flujo de las actividades y las transformaciones que ocurren en el interior del proceso.

\section{Marco teórico}

\subsection{Los servicios}

Los servicios son actividades económicas que producen lugar, tiempo, forma o utilidad psicológica para el cliente (Chiavenato \& Campos, 2012) y también pueden incluir soporte informacional para el cliente, entrega rápida y confiable, instalación del producto, servicio de posventa y solución de problemas. Ejemplificando como cliente interno, el sector de la producción es un cliente del almacenaje. Ya como cliente externo se pudo citar a la compañía encargada de transportar los productos. La identificación clara de los clientes es una etapa importante y esencial en la definición de los servicios deseados. La figura 1 presenta algunas categorías de servicios en esferas diferentes con los actos tangibles e intangibles.

Los procesos deberían posibilitar la visualización y entendimiento de las diversas fases de producción de un bien o servicio. También (Hammer, Champy, \& Korytowski, 2000) presentan el proceso empresarial como un conjunto de actividades que tienen uno o más tipos de entrada, generando una salida de valor para el cliente, o sea, la entrega del producto o servicio al cliente. Cuando se tienen procesos más maduros todos tienen el conocimiento lato, entonces es posible obtener más ganancias sin demandar más inversiones. Proceso, por lo tanto, es una ordenación lógica de actividades interconectadas, cuyo objetivo es producir productos o servicios que representan valor para clientes. Son características esenciales de procesos la regularidad, la previsibilidad del resultado y el conocimiento de las condiciones para su realización (Kim \& Jang, 2002). En la misma línea de consideración (Dávila, Leocádio, \& Varvakis, 2010), se percebe que los procesos empresariales, entendidos como flujos de valor, deberían ser constantemente evaluados o incluso medidos, mejorando y/o recreados para acompañar tanto los cambios en los objetivos como las necesidades de la organización.

De esa manera, (Harrington, 2000) enfatiza que la gestión de los procesos empresariales debe concentrarse tanto en la mejora continua como en las metodologías de modificación, implicando benchmarking, reestructuración del proceso, mejora enfocada, nuevo diseño del flujo del proceso, innovación del proceso, costo basado en la actividad y etc. 


\section{Figura 1}

Tipos de procesos de servicios.

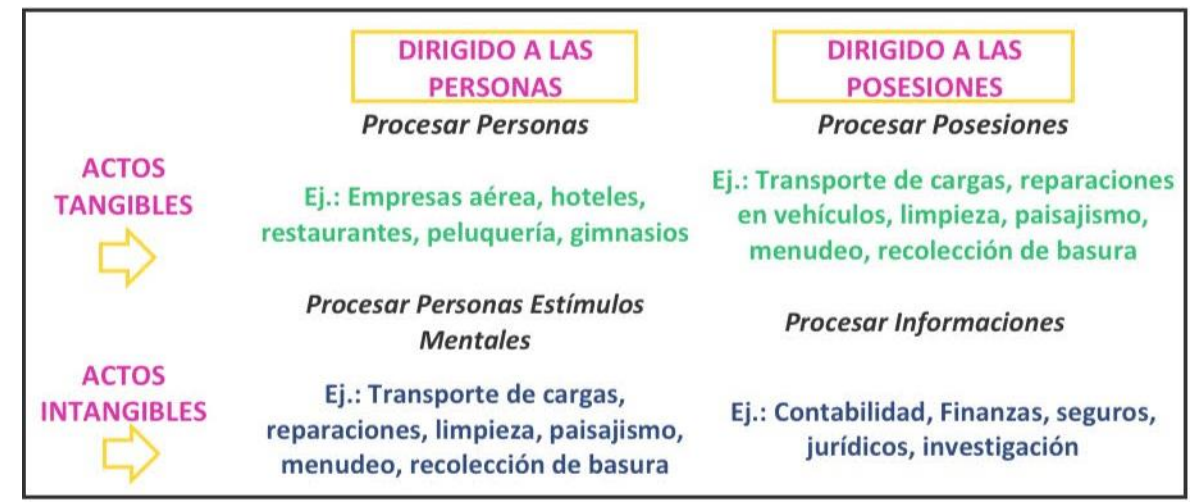

Fuente: (Chistopher \& Wright, 2015).

Autores renombrados (Fitzsimmons \& Fitzsimmons, 2014; Ou, Verhoef, \& Wiesel, 2017; Santos, L C, Gohr, C F, Varvakis, 2011; Zeithaml, Bitner, \& Gremler, 2014) acaban por sintetizar las características que vienen complementar otras y considera los servicios como las siguientes especificidades:

Producción y consumo simultáneos: la producción del servicio sucede al mismo tiempo en que ocurre el consumo. Participación del cliente: se puede afirmar que en los servicios el cliente es quien inicia el proceso de producción, siendo que la participación del cliente es lo que hace disparar el proceso. Sin embargo, según (Santos, L C, Gohr, C F, Varvakis, 2011), el cliente evalúa no solo el resultado, como también el proceso, esto es, la forma en que se ejecuta el servicio.

\subsection{Tipos de procesos en servicios}

Servicios profesionales o personalizados: servicios profesionales presentan un bajo volumen y alta variedad debido a su alto nivel de personalización, que se adapta para satisfacer las necesidades individuales de los clientes. Se basan en personas, con énfasis en el proceso (Slack et al., 2013). Servicios de masa: servicios de masa presentan alto volumen y baja variedad. Son basados en equipamientos con orientación para el producto. Como no es un servicio profesional, debe seguir procedimientos preestablecidos. En algunas organizaciones se conocen como Procedimiento Operativo Estándar SOP (Stuany, de Lima, \& Moreira, 2018).

Tienda de servicios: las tiendas de servicios se encuentran entre los servicios profesionales y los de masa, porque son caracterizadas por nivel de contacto con el cliente, personalización, volumen de clientes y libertad de decisión. Podemos incluir en los servicios profesionales o personalizados otros como, por ejemplo, las concesionarias de ventas de vehículos y los servicios que de ella advén, como se muestra la figura 2, con las divisiones orientadas por el énfasis y grado de variabilidad versus el número de clientes procesados por día. 


\section{Figura 2}

Procesos de Prestación de Servicios

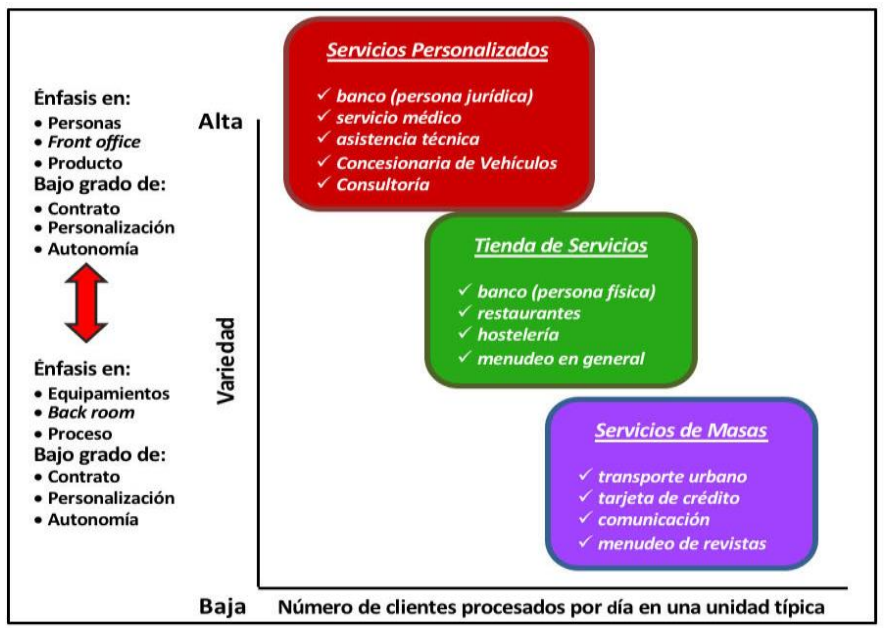

Fuente: (Chistopher \& Wright, 2015).

Sobresale que hay por lo menos cuatro razones (Davenport \& Prusak, 2003) para documentar procesos antes de proponer mejorías, que pueden ser enumeradas aquí: (i) el entendimiento de los actuales procesos que facilita la comunicación entre los gestores de la empresa; (ii) en organizaciones complejas, es difícil pasar para un nuevo proceso sin comprender el actual, porque se puede sobresalir operaciones importantes en el rediseño; (iii) el reconocimiento de problemas existentes en el actual proceso puede ayudar a evitar su repetición en el nuevo proceso; y (iv) el entendimiento de los actuales procesos posibilita mensurar el valor de la reingeniería propuesta.

\subsection{Calidad del Servicio}

En relación con los servicios, la calidad está vinculada a la satisfacción de los clientes y estos se vuelven retos para las empresas, o sea, sus satisfacciones, así como las retenciones son posibles de ocurrir en todas las empresas de varios segmentos distintos. En el caso de las concesionarias, los clientes tienen expectativas sobre la calidad y el nivel de los servicios de posventa que el producto trae consigo (Sviokla \& Shapiro, 1994).

Esto viene al encuentro del valor percibido por el cliente, que es la principal sustentación de los relacionamientos entre una empresa y sus clientes, por objetivar, o mismo representar lo que el cliente entiende, como fundamental para iniciar y mantener una relación de larga data con el proveedor del servicio que incluya la permanencia comprador o consumidor (Zeithaml, Lemon, \& Rust, 2001).

Os servicios de las concesionarias son importantes (Gurgel, Nóbrega, Souza, \& Côrtez, 2011) por representar "el final de la cadena en la que se encuentran fabricantes de componentes, montadoras y comerciantes y que la insatisfacción generada en cualquier parte de esta cadena se reflejará en todos sus componentes" (Borges, Lima, Beber, \& Rossi, 2006, 2013). Dentro de ese pensamiento la calidad 
de los servicios de la concesionaria será un factor preponderante en la fidelización del cliente, evitando así la substitución por un competidor. El Cuadro 1 demuestra los cincos principales factores determinantes de la calidad en los servicios para los consumidores.

Cuadro 1

Principales factores determinantes de calidad en los servicios

\begin{tabular}{|l|l|}
\hline Atributo & Descripción \\
\hline Confiabilidad & $\begin{array}{l}\text { La capacidad de prestar el servicio comprometido de manera confiable y } \\
\text { con precisión }\end{array}$ \\
\hline Sensibilidad & $\begin{array}{l}\text { La disposición para atender y ayudar al consumidor y proporcionar un } \\
\text { servicio rápido }\end{array}$ \\
\hline Seguridad & $\begin{array}{l}\text { El conocimiento y la cortesía de los empleados y sus habilidades en } \\
\text { transmitir confianza }\end{array}$ \\
\hline Empatía & La atención y el cariño individualizados proporcionados al consumidor \\
\hline Tangibilidad & $\begin{array}{l}\text { La apariencia física de las instalaciones, equipos, empleados y materiales } \\
\text { de comunicación }\end{array}$ \\
\hline
\end{tabular}

Fuente: Adaptado (Berry \& Parasuraman, 2004).

Destaca (Grönroos, 2004):

“[...] cuando el desempeño del servicio está por encima de lo esperado, la empresa prestadora de servicio logra encantar o sorprender su cliente, siendo que este evaluará el servicio como de calidad superior. Cuando el nivel de servicio percibido está por debajo de lo que el cliente considera adecuado, hay una discrepancia entre el rendimiento del proveedor y las expectativas de ese cliente".

La conceptúan (Berry \& Parasuraman, 2004; Parasuraman \& Berry, 1991), estas discrepancias y como lagunas o gaps de la calidad, e indica cinco tipos principales:

GAP1: Percepción de la administración - Se refiere a la discrepancia que puede existir entre el deseo real del cliente y el que el gestor se imagina;

GAP2: Especificación de calidad - Se refiere a la discrepancia entre especificaciones de calidad en el servicio y la percepción del gestor;

GAP3: Entrega del servicio - Se refiere a la discrepancia entre el estándar de servicio establecido de acuerdo con el deseo del cliente ya su ejecución;

GAP4: Comunicación de mercado - Discrepancia entre las promesas hechas y lo que es realmente cumplido por las empresas prestadoras de servicio;

GAP5: Calidad percibida de servicio - Discrepancia entre el servicio deseado y el servicio percibido por el cliente. 


\subsection{Asignaciones de procesos y estandarización}

Al iniciar el mapeo, es necesario la definición de la familia de servicios, que es la clasificación de los tipos de servicios realizados en la concesionaria versus el tiempo de realización y cantidad de servicios - con esta definición las agrupaciones de servicios pueden viabilizar se, además del diseño del mapa con foco en determinados tipos de servicios que posibilitan criar trabajo estandarizado.

La gestión de los procesos es una metodología que nos permite maximizar la capacidad de satisfacer y superar los requisitos de los Clientes, alcanzar el resultado esperado de manera más simple y por el menor costo, aumentar la capacidad del proceso de atender los cambios de las necesidades de los Clientes y del negocio, sin reducir la efectividad y la eficiencia del proceso. Observe que la gerencia de procesos es el método por el cual se pueden alcanzar las metas de calidad (Campos, 2016).

La reinvención de los procesos en las empresas dio lugar a una gran oportunidad de desarrollo de enfoques de cambio organizacional. Esta oportunidad de mercado, asociada a la necesidad de las organizaciones existentes, además de la vasta y actual bibliografía del área empresarial, acentuó su interesen los procesos organizacionales y en su importancia para el desenvolvimiento de una organización innovadora y competitiva en ambientes de extrema competitividad, han posibilitado muchas técnicas y herramientas destinadas al mapeo, modelaje y rediseño de procesos.

En este sentido, el mapeo es una herramienta gerencial y analítica de los procesos que posibilitan, la reducción de costos en el desarrollo de productos y servicios, la reducción en las fallas de integración entre sistemas y mejora del desempeño de la organización, además de ser una herramienta que viene a posibilitar el mejor entendimiento de los procesos actuales y eliminar o simplificar aquellos que necesitan de cambios (Villela, 2000). Uno de los puntos relevantes del presente estudio son los principales beneficios resultantes de un mapeo de procesos, ya que permiten una secuencia que estimula la ejecución de cada actividad, generando indicadores gerenciales.

\subsection{Las técnicas de Mapeo}

Por el uso de diferentes técnicas, el mapeo de procesos propone diferentes enfoques, y la exacta interpretación de estas técnicas es de fundamental relevancia. Las técnicas pueden ser utilizadas individualmente o por separado, dependiendo de los procesos que se van a mapear.

La literatura presenta algunas técnicas (Mello, 2008, 2011), de mapeo con diferentes enfoques haciendo la correcta interpretación de estas técnicas fundamental en el proceso de mapeo. Donde podemos citar:

SIPOC: es una herramienta usada por un equipo para identificar todos los elementos pertinentes de un proyecto de mejora de proceso antes que el trabajo empiece (Widi Bhawika \& Suef, 2019). 
Blueprinting: representa un diagrama de flujo de todas las transacciones integrantes del proceso de prestaciones de servicio (Fitzsimmons \& Fitzsimmons, 2014; Silveira, Nogueira, Koefender, Tinoco, \& Amaral, 2019);

Diagrama de Flujo: técnica que permite el registro de acciones de algún tipo y puntos de toma de decisiones que ocurren en el flujo real.

Mapeo de flujo de valor (VSM - (value stream map) según (Barnes, 2001), el flujo de valor es un diagrama de flujo dibujado sobre la planta de un edificio o disposición para visualizar mejor el proceso. O como los recursos y la información disponible ion fluyen por el proceso como outputs e inputs

Diagrama hombre-máquina: se tiene por objetivo el estudio da interrelación entre el trabajo del hombre y el de la máquina, identificando los tiempos ociosos de ambos y balanceando la actividad del puesto de trabajo (Barnes, 2001).

IDEF0 a IDEF9: Diagramas que representan un diseño del comportamiento de los clientes.

Así, se establecieron convenciones para la creación de diagramas de flujo que posibilitaran análisis más eficientes y con menor tiempo de construcción, dando aquí la debida importancia a los procesos computacionales.

En este estudio se utilizaron las herramientas computacionales de diagramación de flujo para análisis del proceso de producción de servicio de la concesionaria, pues, como caracterizado por (Santos, Martins, \& Da Hora, 2019; Villela, 2000), estas herramental "ayudan a definir procesos por la descripción a través de símbolos, sirviendo para dibujar y diagramar flujos".

\subsection{Los GAPs de servicios en la concesionaria}

Los gaps dejados en la realización de los servicios son en su mayoría oportunidades de mejoría que la empresa, a través de sus colaboradores, permiten que sean percibidas por los clientes, cuando dejan o simplemente no realizan actividades dentro de lo esperado por el cliente que son las expectativas.

Argumentan (Kotler, 2000) que los compradores forman sus expectativas basándose en experiencias de compras anteriores, consejos de amigos y colegas, informaciones y promesas de profesionales de marketing y de concurrentes. Es decir, retener clientes pasa a ser no sólo una consigna en la empresa, sino también una estrategia para su continuidad. Entonces:

Los gaps aquí estudiados en este trabajo fueron:

a) Entrega de servicio - Se refiere a la discrepancia entre el estándar de servicio establecido de acuerdo con el deseo del cliente y su ejecución. En este caso, cuando el vehículo es recibido en la concesionaria, un colaborador prescribe, junto con el cliente, el diagnóstico de acuerdo con las 
informaciones colidas por el cliente frente a la ficha de datos del sistema de informaciones de la empresa.

b) Comunicación de mercado - Discrepancia entre las promesas hechas y lo que es realmente cumplido por las empresas prestadoras de servicio. Esta discrepancia deja al cliente descontento y con la sensación que fue lesionado y aun teniendo que pagar por eso. Muy a regañado vuelve a la concesionaria.

c) Calidad percibida de servicio - Discrepancia entre el servicio deseado y el servicio percibido por el cliente. La falta de conocimiento específico del servicio técnico por parte del cliente acerca del trabajo realizado no invalida la percepción del valor cualitativo entre el que se espera como resultado en este caso, la solución definitiva del problema que motivó la demanda por este servicio.

En este contexto, se abren oportunidades y posibilidades para mejoras significativas en los procesos de la concesionaria, a través del estudio de los procesos, tales como identificar los gaps y proponer soluciones que vengan a satisfacer a los clientes; aumentar las ganancias y reducir los desperdicios de tiempo y material involucrado en la consecución de los servicios.

\section{Resultados y discusión}

A referirse a las concesionarias de vehículos, (Crispim, Baptista, Lima, Lopes, \& Medina, 2007) dice que ellas, en Brasil, se caracterizan como reventas autorizadas, que tiene exclusividad de la automotriz. En algunos casos pertenecen a una red de franquías, y solo pueden comprar de la marca a la cual representan y son responsables por la venta al consumidor final. Sin embargo, varias marcas que están disputando el mismo mercado de vehículos nuevos pertenecen, en algunos casos, a un único dueño. Considerando esa situación que es fomentada por la baja rentabilidad en la comercialización de los vehículos nuevos por las concesionarias autorizadas, afirma (Crispim et al., 2007), que estos necesitan compensar las pérdidas de margen con el aumento de volumen y de giro, así como ganar poder de negociación en relación con los proveedores.

En este contexto y con el crecente interés en el estudio de los servicios de forma general, y en este en particular, y el sentimiento expresado por el cliente, que tiende a crecer, a la medida que tal actividad asuma mayor importancia en la economía del país y mundial, se torne necesario una investigación más eficiente por informaciones más robustas y con certeza asertiva.

Como hace notar (Liou, Tsai, Lin, \& Tzeng, 2011) los clientes esperan, es un paso crucial para un servicio de alta calidad. Para la grande parte de los autores, apenas los clientes pueden realmente definir la calidad del servicio. También (Wu \& Lin, 2012), explicitan que las empresas deben entender la importancia del servicio, crear valores para el cliente, escuchar sus voces y hacer la calidad del servicio y satisfacción del cliente su objetivo, se esforzando para satisfacer sus necesidades. 
Con relevancia significativa, entender lo que el cliente espera o mismo desea es esencial en los servicios. Mantiene que el servicio de asistencia técnica (Chistopher \& Wright, 2015) en automóviles o similares es un servicio dirigido a los bienes de las personas, dispensando la presencia de estas, cuya participación se limita en la mayoría de las veces en entregar el bien, explicar el problema y después regresar para recogerlo y pagar la cuenta, siendo que la producción en cada caso debe ser una solución satisfactoria para el problema del cliente o alguna mejora tangible del artículo en cuestión. La única cosa que el cliente sabe es que generalmente tiene un problema o que debe ser hecha una determinada revisión en su vehículo.

Este hecho hace con que algunas concesionarias determinen al cliente que es necesario hacer un diagnóstico para definir lo que debe ser hecho y que, para eso, el vehículo necesita quedarse en la concesionaria el día todo. Esta previsión muchas veces es hecha hasta para una revisión simple y cambio de aceite básico. De hecho, lo que, o cliente desea es recuperar su vehículo lo más pronto posible o de preferencia hasta el final del día o aun conforme la programación inicialmente acordada entre las partes. En este caso en particular, la empresa trabaja con vehículos de flotas, vehículos alternativos (que circulan en la cuidad de forma a complementar la flota de los autobuses de línea) o aun de órganos públicos de las diversas esferas del poder, y que los motoristas se queden por muchas veces en la espera por la liberación de los vehículos para la complementación de sus turnos de trabajo.

En relación a las características, las concesionarias presentan semejanzas genéricas en sus estructuras: Sala de explosión para la comercialización de vehículos nuevos y/o usados (Ventas); Tiendas de piezas, donde son comercializadas las piezas de reposición para clientes externos y para talleres; Departamento de servicios (mantenimiento y preventivo), donde son realizados los servicios de revisión, mantenimiento, garantía, documentación, seguros, accesorios, y otros; Departamento de Calidad; y Posventas (Garantía).

El sector de autobús y minibús presenta una característica común y similar para la satisfacción y mantenimiento de clientes, afirma (Sant'Anna, 2004) o sea: una fuerte dependencia de los servicios de posventa debido a ser casi servicios exclusivos en la ciudad. Esta dependencia tiene aumentado, en los últimos años, en la ciudad por el hecho de que los productos de este segmento tengan solo dos empresas que préstenlos servicios. Entonces, si crear ventajas competitivas en este sector es un desafío, especialmente porque los servicios de posventa continúan siendo percibidos por el cliente, en su mayoría, como un mal necesario. Otro aspecto, aquí levantado se realiza por regla general, en virtud de la imposición de los términos de garantía, o sea, es el uso de los servicios de posventa, para mantenimiento preventivos y correctivos, en concesionarias autorizadas durante la vigencia del período de garantía, que varía de 12 a 24 meses, conforme el fabricante.

Entonces, en este sentido, las empresas necesitan de una evaluación de desempeño organizacional continúa, siendo esta una actividad fundamental en la gestión empresarial en cualquier 
segmento. En esta línea de raciocinio se añaden los indicadores de desempeño, que asumen papel central por representar un instrumento capaz de proporcionar datos que, después de tratados, pueden transformarse en informaciones útiles para el proceso y, con eso, el gestor hará su toma de decisión cada vez más acertada (Sehnem, Kipper, Da Silva, Freitas, \& Choaire, 2020).

La gestión de las micro y pequeñas empresas por medio de indicadores de desempeño no es solamente la administración de pantallas de ordenador, planillas o gráficos, sino, una decisión viable y procedente, con foco en los resultados planeados por la organización.

En general, la evaluación es influenciada directamente por los objetivos y metas de la empresa, que se reflecten en el ejercicio del controle y están correlacionados con las fases de ejecución y planificación de las actividades de la empresa. Durante las prestaciones de servicios de mantenimiento preventivo, ya es posible consolidar una primera estructura de flujo de procesos.

Entre las posibles ganancias en adoptar los indicadores de desempeño y gestión empresarial, se puede citar: entender su negocio buscando identificar y actuar en los riegos que pueden estar comprometiendo la vida de la empresa; garantir la subsistencia del empresario y de sus funcionarios; garantir la facturación de la empresa hoy y en el futuro; conocer los costos operacionales de la empresa; evaluar el grado de satisfacción de los clientes; atender a los plazos de entrega, y mejorar la calidad del producto o servicio.

Las Figuras 3 y 4 a seguir muestran el diagrama de flujo del proceso del área de atención al cliente, debidamente mapeado, que consiste en la gestión dos procedimientos de recepción de los clientes en la empresa. El intento de este proceso es presentar una atención con alto nivel de calidad visando el fortalecimiento en el relacionamiento con el cliente. 
Figura 3

Diagrama del área de atención al cliente

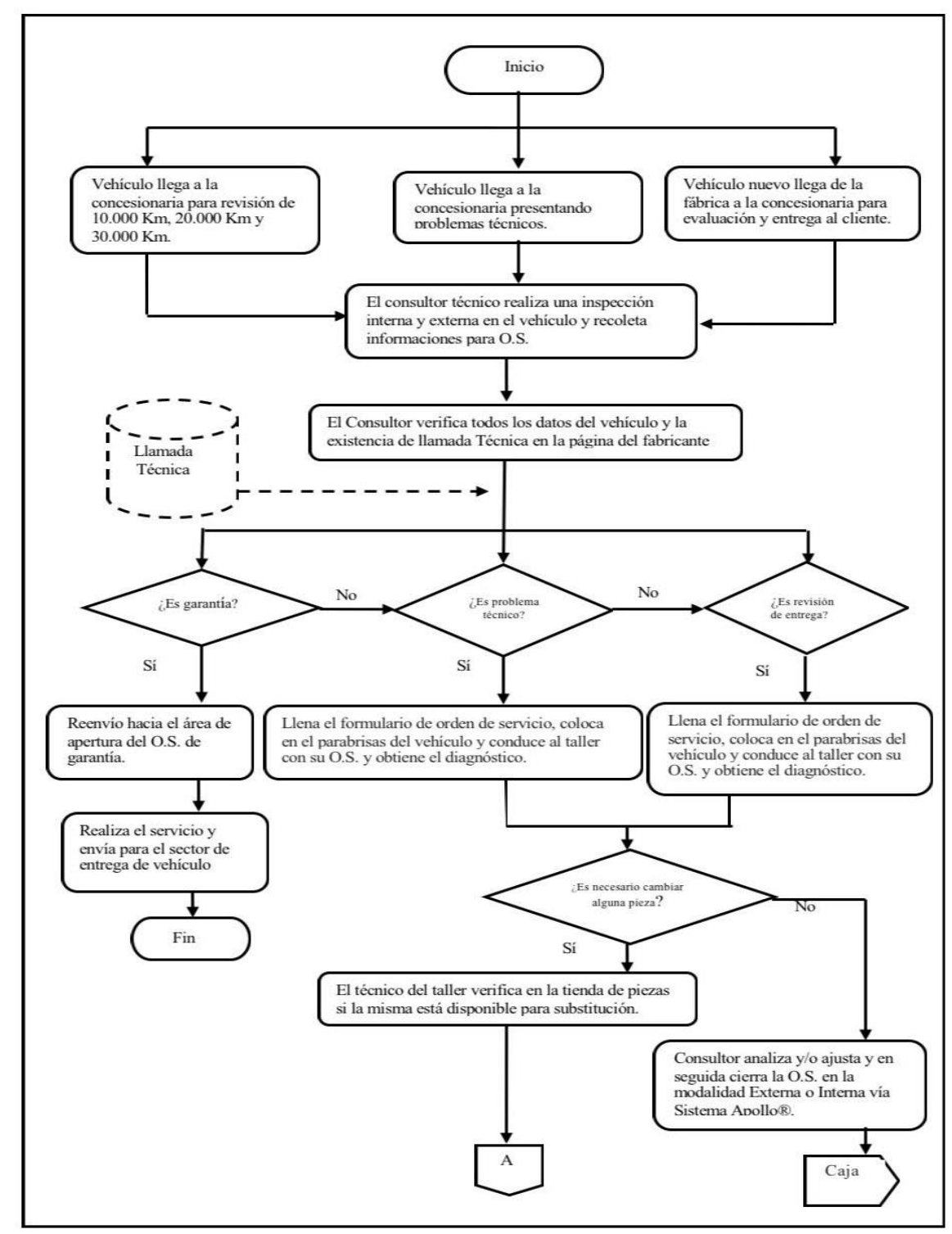

Fuente: Elaboración propia (continuación).
Figura 2

Diagrama del área de atención al cliente

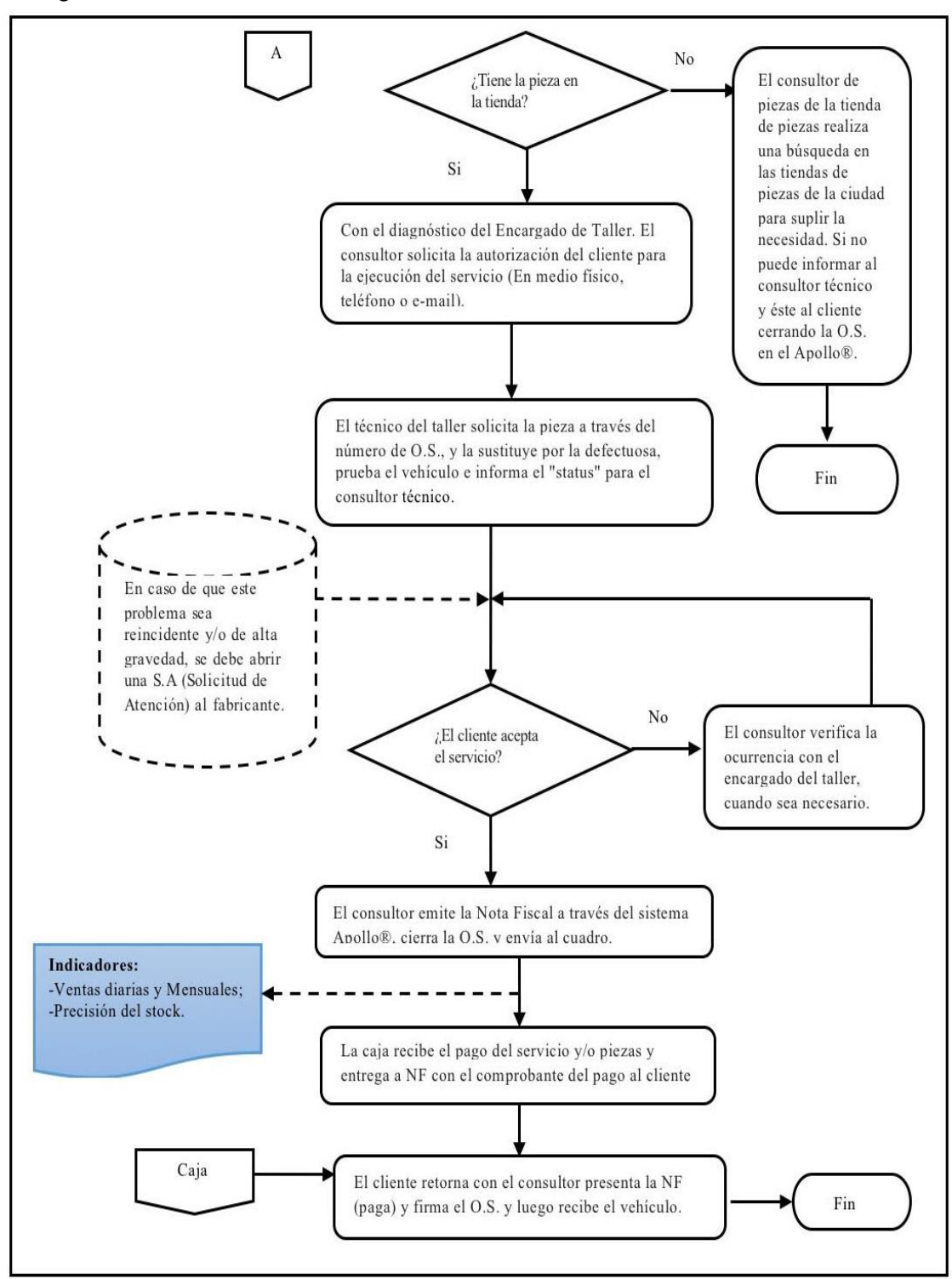

Fuente: Elaboración propia. 
La Figura 5 presenta el total de órdenes de servicio recibidas mensualmente, estratificando la cantidad de servicios, oportunista al gestor entender el proceso más claramente e interviniendo puntual o sistemáticamente en la solución de problemas que incurren en la pérdida de clientes. El indicador también puede establecer la demanda de servicio, teniendo así, como el gestor de evaluar la necesidad de mano de obra necesaria actual y proyectar también la futura.

\section{Figura 4}

Total de las Órdenes de Servicio Recibidas.

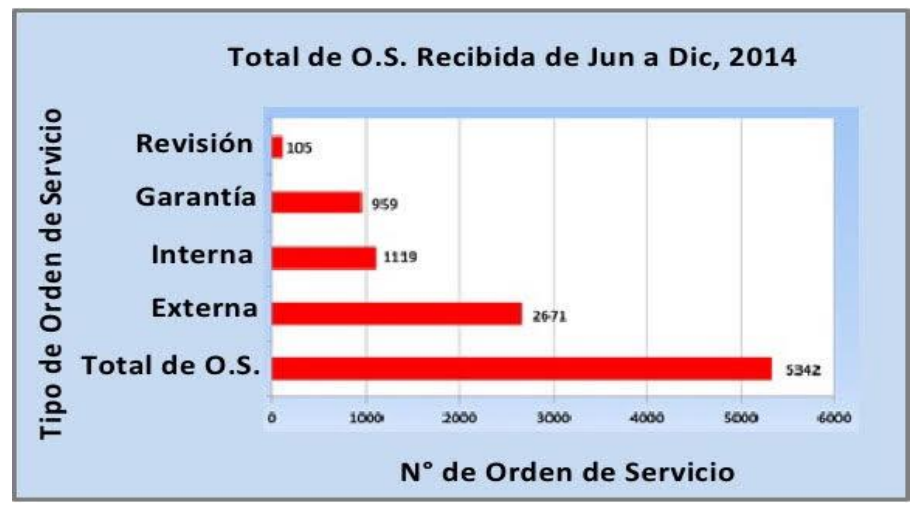

Fuente: Elaboración propia

En cuanto a la atención de la tienda de piezas para clientes externos e internos, se observaron muchos problemas, pues había discrepancias exageradas en el control de la precisión del stock, identificación/localización inexacta de las partes y piezas en el stock y medición del stock de seguridad.

También existe la atención por el paso-piezas (área destinada a la atención exclusiva de los mecánicos y el personal del taller). En este caso, la atención se realiza a través de O.S. para la retirada de las piezas del stock y al final del día se realiza a la baja en la acción. La Orden de Servicio (O.S.) es un documento que formaliza el servicio prestado por la asistencia técnica a un cliente, así como, indica los materiales y la mano de obra a ser utilizados en la ejecución de un determinado servicio. El cliente, en posesión de la documentación, retorna la tienda y en el mostrador de entregas recibe su mercancía y, mediante la conferencia, la Nota Fiscal está sellada con la expresión "MERCANCÍA ENTREGADA" y firmada por el responsable de la entrega de mercancías. Al final de los eventos se introdujo un monitoreo para recolección de datos para input en los indicadores de desempeño.

Después de análisis de las actividades mapeadas surgió la oportunidad de implementar una recolección de datos para generar los indicadores y también la adición en los procesos el conteo cíclico con uso de la metodología o clasificación ABC.

Entonces, todos los días eran informados al almacén de los elementos que debían ser contados, y éstos eran sugeridos por una herramienta del software Apollo ${ }^{\circledR}$. Los ítems eran contactos físicamente y comparados con el sistema contable. Los resultados divergentes o correctos se añadían al medidor de 
la exactitud de la acción. La figura 6 a continuación presenta la precisión de la acción medida diariamente e informada a todos mensualmente, es posible observar los valores medidos no alcanzan las metas que fueron planificadas de forma creciente y gradualmente a lo largo de los meses medidos. Sólo el mes de octubre de 2014 alcanzó la meta establecida. Es posible percibir que estos resultados debilitan los controles y pone en riesgo la confianza de la disponibilidad de las partes y piezas existentes en el stock.

\section{Figura 5}

Precisión del inventario medido.

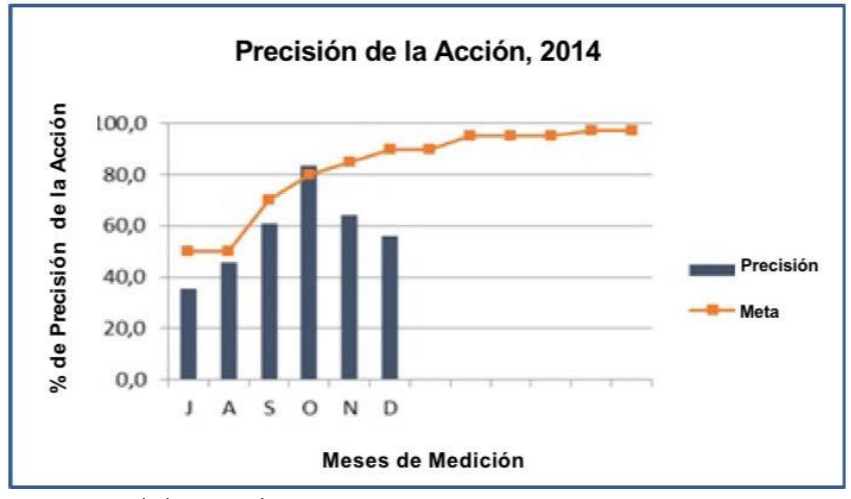

Fuente: Elaboración propia.

El indicador también posibilita la visualización de posibles errores en las compras de reposición del stock, adquisiciones indebidas o en duplicidad además de causar errores en la venta de los productos.

\section{Conclusiones}

El estudio buscó presentar la importancia de los análisis a través de diagramas de procesos de servicios, como técnica para el mapeo de procesos en la concesionaria de microbuses, "alfa" desde la recepción del vehículo hasta su entrega al cliente, buscando así eliminar posibles gaps.

A lo largo de los análisis, se verificaron posibilidades de inserción de los puntos de monitoreo a través de los indicadores de desempeño. Esto indica que todos los procesos pueden y deben ser asignados, tanto los de contacto directo con el cliente como los otros procesos adyacentes, incluso aquellos donde los clientes no tienen acceso. Aunque los resultados obtenidos demuestran la necesidad de controles a lo largo de los procesos de la concesionaria, queda claro que los mismos sólo pudieron ser verificados, y secuencialmente tratados, cuando se iniciaron las entrevistas con los funcionarios de acuerdo con los apéndices y, consecuentemente, la elaboración de los resultados, que permitió orientar 
a los colaboradores sobre la secuencia de actividades a ser ejecutadas, incrementar servicios de calidad, promoviendo la integración de los equipos y clientes y luego finalizado por los análisis, culminando con la implantación de los indicadores de desempeño de la empresa en sus procesos.

El estudio se inició con el levantamiento del proceso actual, identificación de los requisitos del proceso, conocimiento del proceso, la confección de los diagramas de flujo y por último los análisis para la implantación de los indicadores de desempeño. En la empresa objeto del estudio se buscó analizar posibles "gaps" involucrados en la realización del servicio en los procesos de mantenimiento preventivo y correctivo aplicados en los microbuses, involucrando el taller y la tienda piezas con el uso de la herramienta de mapeo de procesos que fueron discutidas en las debidas secciones de esta investigación. Para que el objetivo general se alcanzara, fueron necesarios objetivos específicos como: analizar los procesos de los servicios del taller y tienda de piezas de la concesionaria, Identificar puntos vulnerables para la realización de los servicios en las empresas e Indicar posibles mejoras para aumentar la eficiencia de los servicios.

Se analizaron las dimensiones de la entrega de servicio, de la Comunicación con el cliente y de la Calidad percibida de servicio discutido en este ítem fueron asignadas todas las actividades en el diagrama de flujo del proceso desde la entrada del vehículo pasando por la realización de las manutenciones hasta la entrega. En otras palabras, cuando los vehículos regresan del taller con el status de "realizado", o sea, el servicio de mantenimiento preventivo y/o correctivo realizados, los mismos son destinados para ser lavados y entregados a sus propietarios. En el momento de la entrega se comprueba lo que se acordó con el cliente, el estándar de servicio, cuando éste dejó en la concesionaria el vehículo, y su deseo de lo que iba a ser realizado, pero muchas veces los servicios no están a contento por parte del cliente. Por lo tanto, se tiene en este ítem la búsqueda por la concordancia del cliente, por más que el cliente en algunas veces no venga a tener el conocimiento técnico para el juicio. Este fue un gap encontrado que, después del mapeo y confección de los diagramas de flujo se percibió: fallas en el tipo de servicio a ser realizado, plazo de entrega, cobranza inadecuada de mantenimiento y falta de material en la tienda (aceite lubrificante y filtros de aire / aceite) como base que muchas veces postergaban la entrega del producto. A lo largo del mapeo se identificaron varios gaps que hacían los servicios vulnerables para el logro de las metas de entrega de los vehículos, como la comunicación con los clientes, el retraso entre la solicitud y confirmación para la realización de los servicios, retorno de vehículos presentando el mismo defecto, retirada de partes y piezas del fabricante.

En esta investigación no se midió la percepción del cliente en cuanto a la calidad del servicio, o sea, el gap entre el servicio deseado por el cliente o sea la satisfacción.

Como característica relevante del método propuesto, la utilización de diagrama de flujo para el mapeo de los procesos ayudó a comprender el proceso y posibilitando una visión más amplia del asunto. Al abordar las posibles mejoras para aumentar la eficiencia de los servicios, el mapeo de los procesos 
fue considerado un factor esencial para esta actividad, pues permite al cuerpo gestor comprender el proceso y de forma ágil tomar acciones puntuales para la mejora continua de los procesos. También, permite valorizar actividades para la mejora del nivel de servicio al cliente. El método de análisis por diagrama de flujo de los gaps propuestos fue considerado adecuado para la aplicación en la concesionaria u otras empresas de servicio de éste o de otros segmentos, entendido por los gestores de los procesos por comprender la facilidad del mismo. Además, de forma asertiva el método de análisis de procesos (mapeo) por diagrama de flujo tiende a ser de fácil visualización por los gestores, además de mejorar su gestión aumentando su participación de la empresa en el mercado en que está inserta.

Por lo tanto, durante el trabajo fue posible levantar e identificar los gaps en los procesos estudiados y analizar sus causas y efectos, sugiriendo mejoras con la implantación de indicadores de desempeño de los procesos y monitorear adecuadamente.

\section{Agradecimientos}

Este trabajo fue una Investigación realizó en una empresa de venta de microbuses y comercializa piezas de repuesto en la ciudad de Manaus, Amazonas - Brasil.

\section{Referencias}

Barnes, R. (2001). Estudo de Movimentos e Tempos, tradução da 6 ed. Americana Por Sérgio LO Assis. Edgar Blücher, Edusp.

Berry, L. L., \& Parasuraman, A. (2004). Marketing services: Competing through quality. Simon and Schuster.

Borges, F. Q., Lima, M. A. S., Beber, S. J. N., \& Rossi, C. A. V. (2006). Estudo da insatisfação do consumidor nos serviços prestados por assistências técnicas autorizadas de automóveis. Perspectivas Contemporâneas, 10(1), 33-49. https://doi.org/doi.org/10.1590/S141565552006000200003

Borges, F. Q., Lima, M. A. S., Beber, S. J. N., \& Rossi, C. A. V. (2013). Insatisfação do consumidor: uma análise nos serviços prestados por concessionárias de energia elétrica. Perspectivas Contemporâneas, 8(1), 33-49.

Campos, J. P. (2016). Mapeamento de processos: uma estratégia vencedora.

Chiavenato, I., \& Campos, E.-. (2012). Princípios da administração: o essencial em teoria geral da administração (2nd ed.). São Paulo: Ed Elsevier - Campos.

Chistopher, L., \& Wright, L. (2015). Serviços: marketing e gestão. São Paulo: Saraiva.

Crispim, S. F., Baptista, J. A. A., Lima, M. A., Lopes, M. S., \& Medina, R. M. (2007). Concessionárias de Veículos: Estratégia de Agregação de Valor por Meio de Serviços. Seminários Em Administração-SemeAd, São Paulo: FEA-USP. 
Davenport, T. H., \& Prusak, L. (2003). Conhecimento empresarial: como as empresas gerenciam seu capital intelectual ( $15^{\circ} \mathrm{ed}$.). Rio de Janeiro: Campus.

Dávila, G. A., Leocádio, L., \& Varvakis, G. (2010). Inovação e Gerenciamento de Processos: Uma análise baseada na Gestão do Conhecimento. Pesquisa Brasileira Em Ciência Da Informação E Biblioteconomia, 3(2).

Fitzsimmons, J. A., \& Fitzsimmons, M. J. (2014). Administração de Serviços-: Operações, Estratégia e Tecnologia da Informação. Amgh Editora.

Gil, A. C. (2017). Como elaborar projetos de pesquisa (6ª Edição). São Paulo: Editora Atlas.

Grönroos, C. (2004). Marketing: gerenciamento e serviços. Elsevier/Campus.

Gurgel, J. C., Nóbrega, K. C., Souza, T., \& Côrtez, A. M. S. (2011). Elementos da Recuperação de Serviços: Um estudo em concessionárias de automóveis. Anais Do XXXVI Encontro Do ANPAD. Rio de Janeiro/RJ, 22.

Hammer, M., Champy, J., \& Korytowski, I. (2000). Reengenharia: revolucionando a empresa em função dos clientes, da concorrência e das grandes mudanças da gerência. Campus Rio de Janeiro.

Harrington, H. J. (2000). Aperfeiçoando processos empresariais: estratégia revolucionária para o aperfeiçoamento da qualidade, da produtividade e da competitividade. São Paulo, MakronBooks.

IBEGE. (2017). Estatísticas brasileiras. Retrieved from http://brasilemsintese.ibge.gov.br/servicos.html Acessado em 21.08.2018.

Kim, S.-H., \& Jang, K.-J. (2002). Designing performance analysis and IDEFO for enterprise modelling in BPR. International Journal of Production Economics, 76(2), 121-133.

Kotler, P. (2000). Administração de marketing (15). São Paulo: Prentice Hall.

Liou, J. J. H., Tsai, C.-Y., Lin, R.-H., \& Tzeng, G.-H. (2011). A modified VIKOR multiple-criteria decision method for improving domestic airlines service quality. Journal of Air Transport Management, $17(2), 57-61$.

Mello, A. E. N. S. (2008). Aplicação do mapeamento de processos e da simulação no desenvolvimento de projetos de processos produtivos.

Mello, A. E. N. S. (2011). Aplicação do Mapeamento de Processo e da simulação no desenvolvimento de projetos de processos produtivos. Itajubá: UNIFEI.

Miyashita, P. T., \& Salomom, V. A. P. (2011). Mapeamento de processos em empresa prestadora de serviços de seguros. Encontro Nacional de Engenharia de Produção, 31.

Ou, Y.-C., Verhoef, P. C., \& Wiesel, T. (2017). The effects of customer equity drivers on loyalty across services industries and firms. Journal of the Academy of Marketing Science, 45(3), 336-356.

Parasuraman, A., \& Berry, L. L. (1991). Marketing services: competing through quality. New York. 
Sant'Anna, Á. L. de M. (2004). Fidelização do cliente: proposta de um modelo de referência para planejamento e avaliação de programas de fidelidade no setor de pós-venda: caso de uma concessionária de caminhões e ônibus (Universidade Federal do Rio Grande do Sul. Escola de Engenharia. Mestrado Profissionalizante em Engenharia.). Retrieved from http://hdl.handle.net/10183/5166

Santos, L C, Gohr, C F, Varvakis, G. (2011). Um método para identificação e análise de competências e recursos estratégicos das operações de serviços. Gestão \& Produção, 18(4), 791-808.

Santos, A. C. S. G. R., Martins, I., \& Da Hora, H. R. M. (2019). Indicador OEE e ferramentas da qualidade: uma aplicação integrada no processo de destilação de uma indústria de biotecnologia. Exacta, 17(2), 165-184. https://doi.org/10.5585/exactaep.v17n2.8183

Sehnem, E. H., Kipper, L. M., Da Silva, J. I., Freitas, F. De, \& Choaire, G. T. (2020). Utilização dos princípios da manufatura enxuta e ferramenta de mapeamento de fluxo de valor para a identificação de desperdícios no estoque de produto acabado. Exacta, 18(1), 165-184. https://doi.org/10.5585/exactaep.v18n1.8629

Silveira, F. da, Nogueira, C. T., Koefender, A., Tinoco, M. A. C., \& Amaral, F. G. (2019). Análise dos Serviços de um Restaurante Fast-Casual por meio de Estratégias de Operações. Produto \& Produção, 19(4). https://doi.org/10.22456/1983-8026.92124

Slack, N., Chambers, S., Johnston, R., \& Betts, A. (2013). Gerenciamento de operações e de processos. Tradução de Sandra de Oliveira. São Paulo, Brasil: Porto Alegre: Bookman.

Stuany, R. N. B., de Lima, T. M., \& Moreira, E. G. (2018). Processo de elaboração de descrição de cargos e mapeamento de processos de uma empresa de fabricação de equipamentos de áudio. Revista Eletrônica Ciências Empresarias, 7(11), 50-61.

Sviokla, J. J., \& Shapiro, B. P. (1994). Mantendo clientes. São Paulo: Makron.

Triviños, A. N. S. (2013). Bases teórico-metodológicas da pesquisa qualitativa em ciências sociais: idéias gerais para a elaboração de um projeto de pesquisa - Reimpressão (Vol. 1). Editora Atlas.

Villela, C. da S. S. (2000). Mapeamento de processos como ferramenta de reestruturação e aprendizado organizacional.

Widi Bhawika, G., \& Suef, M. (2019). Acceleration for the Administration Process of Project Contract in Power Plant Service Company using DMAIC. Journal of Physics: Conference Series, 1175, 012196. https://doi.org/10.1088/1742-6596/1175/1/012196

Wu, H.-Y., \& Lin, H.-Y. (2012). A hybrid approach to develop an analytical model for enhancing the service quality of e-learning. Computers \& Education, 58(4), 1318-1338.

Yin, R. K., \& Grassi, T. D. (2010). Estudo de caso - Planejamento e métodos (4 edição, Vol. 1). Bookman.

Zeithaml, V. A., Bitner, M. J., \& Gremler, D. D. (2014). Marketing de Serviços-: A Empresa com Foco no Cliente. AMGH Editora.

Zeithaml, V. A., Lemon, K. N., \& Rust, R. T. (2001). Driving customer equity: How customer lifetime value is reshaping corporate strategy. Simon and Schuster. 